UWThPh-1996-44

HEPHY-PUB 650/96

TGU-18

ITP-SU-96/03

MADPH-96-952

hep-ph/9607388

July, 1996

\title{
Impact of squark pair decay modes on search for supersymmetric neutral Higgs bosons
}

\author{
A. Bartl,${ }^{1}$ H. Eberl,${ }^{2}$ K. Hidaka,${ }^{3}$ T. Kon, ${ }^{4}$ \\ W. Majerotto ${ }^{2}$ and Y. Yamada ${ }^{5}$ \\ ${ }^{1}$ Institut für Theoretische Physik, Universität Wien, A-1090 Vienna, Austria \\ ${ }^{2}$ Institut für Hochenergiephysik der Österreichischen Akademie der Wissenschaften, \\ A-1050 Vienna, Austria \\ ${ }^{3}$ Department of Physics, Tokyo Gakugei University, Koganei, Tokyo 184, Japan \\ ${ }^{4}$ Faculty of Engineering, Seikei University, Musashino, Tokyo 180, Japan \\ ${ }^{5}$ Department of Physics, University of Wisconsin, Madison, WI 53706, USA
}

\begin{abstract}
We present a detailed study of the decays of the neutral Higgs bosons $H^{0}$ and $A^{0}$ within the Minimal Supersymmetric Standard Model. We find that the supersymmetric modes $\tilde{t} \bar{t}$ and $\tilde{b} \tilde{b}$ can dominate the $H^{0}$ and $A^{0}$ decays in a wide range of the model parameters due to large Yukawa couplings and mixings of $\tilde{t}$ and $\tilde{b}$. Compared to the conventional modes $t \bar{t}$ and $b \bar{b}$, these modes have very distinctive signatures. This could have a decisive impact on the neutral Higgs boson searches at future colliders.
\end{abstract}


The existence of two or more Higgs bosons would be a clear indication that the Standard Model must be extended. For example, the Minimal Supersymmetric Standard Model (MSSM) 四 with two Higgs doublets predicts the existence of five physical Higgs bosons $h^{0}, H^{0}, A^{0}$, and $H^{ \pm}$[2, [3]. In searching for the Higgs bosons it is necessary to study not only the production mechanism, but also all their possible decay modes. Their decays to supersymmetric (SUSY) particles could be very important if they are kinematically allowed. This is indeed the case for the charged Higgs boson $H^{+}$. If all SUSY particles are very heavy, the $H^{+}$decays dominantly into $t \bar{b}$; the decays $H^{+} \rightarrow \tau^{+} \nu$ and/or $H^{+} \rightarrow W^{+} h^{0}$ are dominant below the $t \bar{b}$ threshold [2, [4]. In refs. [5, [6] all decay modes of $H^{+}$including the SUSY-particle modes were studied in the the case that the SUSY-particles are relatively light ; it was shown that the SUSY decay mode $H^{+} \rightarrow \tilde{\tilde{t}} \bar{b}$ can be dominant in a large region of the MSSM parameter space due to large $t$ and $b$ quark Yukawa couplings and large $\tilde{t}$ and $\tilde{b}$-mixings, and that this could have a decisive impact on $H^{+}$searches at future colliders. In this paper we extend this study to the decay of the neutral Higgs bosons $H^{0}$ and $A^{0}$.

The lighter stop $\tilde{t}_{1}$ can be much lighter than the other squarks and even lighter than the $t$ quark due to large $\tilde{t}_{L}-\tilde{t}_{R}$ mixing being proportional to the large top Yukawa coupling $h_{t}$ and the $\tilde{t}$-mixing parameters $A_{t}$ and $\mu$ [7]. Similarly, the lighter sbottom $\tilde{b}_{1}$ can also be much lighter than the other squarks [5]. In the case of large $\tilde{t}$ - and $\tilde{b}$ - mixings one also expects the couplings of $H^{0} \tilde{t} \tilde{t}, A^{0} \tilde{t} \tilde{t}, H^{0} \tilde{b} \tilde{b}$ and $A^{0} \tilde{b} \tilde{b}$ to be large. Here we show explicitly that the modes $\tilde{\tilde{t}} \overline{\tilde{t}}$ and $\tilde{b} \bar{b}$ can indeed dominate the $H^{0}$ and 
$A^{0}$ decays in a wide range of the MSSM parameters.

First we summarize current experimental limits on the squark masses. The D $\emptyset$ group at FNAL [8, 9] obtained mass bounds for squarks and gluinos from the negative search for them. However, they obtained no mass bound for the mass-degenerate five flavors of squarks $(\tilde{q})$ (other than the stop $(\tilde{t}))$ within the MSSM in case the lightest neutralino $\tilde{\chi}_{1}^{0}$ (assumed to be the lightest SUSY particle (LSP)) is heavier than $70 \mathrm{GeV}$; this is mainly due to the massive LSP effect. On the other hand, the LEP-I experiments set the general bound on the $\tilde{q}$ mass $m_{\tilde{q}} \gtrsim 45 \mathrm{GeV}$ [10, 11]. Experiments dedicated to $\tilde{t}$ search at LEP1.5 [11] and Tevatron [12 have set some limits on the lighter stop $\left(\tilde{t}_{1}\right)$ mass for relatively light $\tilde{\chi}_{1}^{0}$, e.g., typically $m_{\tilde{t}_{1}} \gtrsim 55 \mathrm{GeV}$ (for no $\tilde{t}-$ mixing) [11] and $m_{\tilde{t}_{1}} \gtrsim 100 \mathrm{GeV}$ [12], respectively. However, they set no limit on $m_{\tilde{t}_{1}}$ for $m_{\tilde{\chi}_{1}^{0}} \gtrsim 50 \mathrm{GeV}$ 11, 12].

In the MSSM the properties of the charginos $\tilde{\chi}_{i}^{ \pm}(i=1,2)$ and neutralinos $\tilde{\chi}_{j}^{0}$ $(j=1, \cdots, 4)$ are completely determined by the parameters $M, \mu$ and $\tan \beta=v_{2} / v_{1}$, assuming $M^{\prime}=(5 / 3) \tan ^{2} \theta_{W} M$. Here $M\left(M^{\prime}\right)$ is the $\mathrm{SU}(2)(\mathrm{U}(1))$ gaugino mass, $\mu$ is the higgsino mass parameter, and $v_{1}\left(v_{2}\right)$ is the vacuum expectation value of the Higgs $H_{1}^{0}\left(H_{2}^{0}\right)$ [1]. Here $m_{\tilde{\chi}_{1}^{+}}<m_{\tilde{\chi}_{2}^{+}}$and $m_{\tilde{\chi}_{1}^{0}}<\cdots<m_{\tilde{\chi}_{4}^{0}}$. To specify the squark sector additional (soft SUSY breaking) parameters $M_{\tilde{Q}}, M_{\tilde{U}}, M_{\tilde{D}}$ (for each generation) and $A$ (for each flavor) are necessary. The mass matrix for stops in the base of $\left(\tilde{t}_{L}, \tilde{t}_{R}\right)$ reads $[7$, ,

$$
\mathcal{M}_{\tilde{t}}^{2}=\left(\begin{array}{cc}
m_{\tilde{t}_{L}}^{2} & a_{t} m_{t} \\
a_{t} m_{t} & m_{\tilde{t}_{R}}^{2}
\end{array}\right)
$$


with

$$
\begin{aligned}
m_{\tilde{t}_{L}}^{2} & =M_{\tilde{Q}}^{2}+m_{t}^{2}+m_{Z}^{2} \cos 2 \beta\left(I_{t}^{3}-e_{t} \sin ^{2} \theta_{W}\right), \\
m_{\tilde{t}_{R}}^{2} & =M_{\tilde{U}}^{2}+m_{t}^{2}+m_{Z}^{2} \cos 2 \beta e_{t} \sin ^{2} \theta_{W}, \quad \text { and } \\
a_{t} m_{t} & =m_{t}\left(A_{t}-\mu \cot \beta\right) .
\end{aligned}
$$

For the $\tilde{b}$ system analogous formulae hold but with $M_{\tilde{U}}^{2}$ replaced by $M_{\tilde{D}}^{2}$ in eq.(3), and instead of eq.(4), $a_{b} m_{b}=m_{b}\left(A_{b}-\mu \tan \beta\right) . \tilde{b}_{L}-\tilde{b}_{R}$ mixing may be important for large $A_{b}, \mu$ and $\tan \beta$. The slepton sector is fixed by adding (soft SUSY breaking) parameters $M_{\tilde{L}}$ and $M_{\tilde{E}}$.

The masses and couplings of the Higgs bosons $H^{ \pm}, H^{0}, h^{0}$ and $A^{0}$, including leading Yukawa corrections, are fixed by $m_{A^{0}}, \tan \beta, m_{t}, M_{\tilde{Q}}, M_{\tilde{U}}, M_{\tilde{D}}, A_{t}, A_{b}$ and $\mu$. $H^{0}\left(h^{0}\right)$ and $A^{0}$ are the heavier (lighter) CP-even and CP-odd neutral Higgs bosons, respectively. For the Yukawa corrections to the $h^{0}$ and $H^{0}$ masses and their mixing angle $\alpha$ we use the formulae of ref. 13. We include also the potentially large Yukawa corrections to the triple Higgs vertices of $H^{0} h^{0} h^{0}, H^{0} A^{0} A^{0}$ and $h^{0} A^{0} A^{0}$ using the formulae of ref. 14.

In the following, we take for simplicity $M_{\tilde{Q}}=M_{\tilde{U}}=M_{\tilde{D}}$ (for the third generation), $M_{\tilde{L}}=M_{\tilde{E}}=M_{\tilde{Q}}$, and $A_{t}=A_{b}=A_{\tau} \equiv A$. Thus we have as free parameters $m_{A^{0}}, M, \mu, \tan \beta, M_{\tilde{Q}}$ and $A$.

We calculate the widths of all possibly important modes of $H^{0}$ and $A^{0}$ decays: (i) $H^{0} \rightarrow t \bar{t}, b \bar{b}, c \bar{c}, \tau^{-} \tau^{+}, W^{+} W^{-}, Z^{0} Z^{0}, h^{0} h^{0}, A^{0} A^{0}, W^{ \pm} H^{\mp}, Z^{0} A^{0}, \tilde{t}_{i} \overline{\tilde{t}}_{j}, \tilde{b}_{i} \overline{\tilde{b}}_{j}, \tilde{\ell}_{i}^{-} \tilde{\ell}_{j}^{+}$, $\tilde{\nu}_{\ell} \overline{\tilde{\nu}}_{\ell}(\ell=e, \mu, \tau), \tilde{\chi}_{i}^{+} \tilde{\chi}_{j}^{-}, \tilde{\chi}_{k}^{0} \tilde{\chi}_{l}^{0}$, and (ii) $A^{0} \rightarrow t \bar{t}, b \bar{b}, c \bar{c}, \tau^{-} \tau^{+}, Z^{0} h^{0}, \tilde{t}_{1} \overline{\tilde{t}}_{2}, \tilde{t}_{2} \overline{\tilde{t}}_{1}, \tilde{b}_{1} \overline{\tilde{b}}_{2}$, 
$\tilde{b}_{2} \overline{\tilde{b}}_{1}, \tilde{\tau}_{1}^{-} \tilde{\tau}_{2}^{+}, \tilde{\tau}_{2}^{-} \tilde{\tau}_{1}^{+}, \tilde{\chi}_{i}^{+} \tilde{\chi}_{j}^{-}, \tilde{\chi}_{k}^{0} \tilde{\chi}_{l}^{0}$. Formulae for these widths are found in ref.[2]. In principle, also the decays $H^{0} \rightarrow \tilde{q}_{\alpha} \overline{\tilde{q}}_{\alpha}(q=u, d, c, s$ and $\alpha=L, R)$ could contribute via their gauge couplings. As the squarks of the first two generations are supposed to be heavy, these decays will be strongly phase-space suppressed. Even if they were kinematically allowed, they would have a rate at most comparable to that of $H^{0} \rightarrow$ $\tilde{\ell}_{i}^{-} \tilde{\ell}_{j}^{+}$and $\tilde{\nu}_{\ell} \overline{\tilde{\nu}}_{\ell}$ (see fig. 2 below). We neglect loop induced decay modes (such as $H^{0} \rightarrow g g$ and $\gamma \gamma$ ) and three-body decay modes [15].

In order not to vary many parameters, in the following we fix $m_{t}=180 \mathrm{GeV}$ and $\mu=300 \mathrm{GeV}$, and take the values of $M$ and $\tan \beta$ such that $m_{\tilde{\chi}_{1}^{0}} \simeq 70 \mathrm{GeV}$ for which the $\mathrm{D} \emptyset$ bounds on $m_{\tilde{q}}$ and $m_{\tilde{t}_{1}}$ [8, 12] and the LEP1.5 bound on $m_{\tilde{t}_{1}}$ [11] disappear.

In fig. 1 the contour lines for the branching ratios $B(H \rightarrow \tilde{\tilde{t}}, \tilde{\tilde{b}} \tilde{\tilde{b}}) \equiv$ $\sum_{i, j=1,2}\left(B\left(H \rightarrow \tilde{t}_{i} \overline{\tilde{t}}_{j}\right)+B\left(H \rightarrow \tilde{b}_{i} \overline{\tilde{b}}_{j}\right)\right)\left(H=H^{0}\right.$ or $\left.A^{0}\right)$ are plotted in the $A-M_{\tilde{Q}}$ plane for $\left(m_{A^{0}}(\mathrm{GeV}), M(\mathrm{GeV}), \tan \beta\right)=(450,160,2)(\mathrm{a}),(500,160,2)(\mathrm{b}),(450,146,12)(\mathrm{c})$ and $(500,146,12)(\mathrm{d})$, for which $m_{\tilde{\chi}_{1}^{+}}(\mathrm{GeV})=128,128,131$ and 131, respectively. In the plots we have required $m_{\tilde{t}_{1}, \tilde{b}_{1}, \tilde{\ell}}>m_{\tilde{\chi}_{1}^{0}}(\simeq 70 \mathrm{GeV})$. In the allowed regions of fig.1 we find $m_{H^{0}} \simeq m_{A^{0}}$ and $m_{h^{0}}>55 \mathrm{GeV}$, which satisfies the LEP limit $m_{h^{0}}>44 \mathrm{GeV}$ [16]. We see that the branching ratios $B\left(H^{0} \rightarrow \tilde{\tilde{t}}, \tilde{b} \tilde{\tilde{b}}\right)$ and $B\left(A^{0} \rightarrow \tilde{\tilde{t}}, \tilde{b} \tilde{\tilde{b}}\right)$ can be larger than $50 \%$ in a sizable region. In this region, these SUSY decay modes dominate over the conventional modes.

In fig. 2 we show the $m_{A^{0}}$ dependence (in the $m_{A^{0}}$ range for $m_{h^{0}}>50 \mathrm{GeV}$ ) of the important branching ratios of $H^{0}$ and $A^{0}$ decays for $\left(M_{\tilde{Q}}(\mathrm{GeV}), A(\mathrm{GeV})\right.$, 
$M(\mathrm{GeV}), \tan \beta)=(102,325,160,2)(\mathrm{a}, \mathrm{b}),(218,405,145,30)(\mathrm{c})$, and $(145,274$,

$146,12)(\mathrm{d})$. In these three cases we have $\left(m_{\tilde{t}_{1}}, m_{\tilde{t}_{2}}, m_{\tilde{b}_{1}}, m_{\tilde{b}_{2}}, m_{\tilde{\chi}_{1}^{+}}\right)=(100,270$, 100, 115, 128) GeV (a, b), (80, 386, 80, 304, 132) GeV (c), and (80, 310, 80, 199, 131)

$\mathrm{GeV}(\mathrm{d})$. We see that in these cases the sum of the $\tilde{\tilde{t}}$ and $\tilde{b} \tilde{\tilde{b}}$ modes dominates the $H^{0}$ and $A^{0}$ decays in a wide range of $m_{A^{0}}$. Here note that $m_{H^{0}} \simeq m_{A^{0}}$ in the $m_{A^{0}}$ range shown here, and that the $A^{0}$ does not couple to $\tilde{t}_{i} \overline{\tilde{t}}_{i}, \tilde{b}_{i} \overline{\tilde{b}}_{i}$ and $\tilde{\tau}_{i}^{+} \tilde{\tau}_{i}^{-}(i=1,2)$.

As for $h^{0}$ decay, we have found that the decay $h^{0} \rightarrow \tilde{t}_{1} \overline{\tilde{t}}_{1}$ is kinematically allowed only in a very limited region of the MSSM parameter space.

The reason for the dominance of the $\tilde{\tilde{t}}$ and $\tilde{b} \tilde{\tilde{b}}$ modes in the $H^{0}$ decay is as follows : The modes $t \bar{t}, b \bar{b}, \tilde{\tilde{t}}$ and $\tilde{b} \tilde{\tilde{b}}$ (whose couplings to $H^{0}$ are essentially $\sim h_{t} \sin \alpha$, $\sim h_{b} \cos \alpha, \sim(A-\mu \cot \alpha) h_{t} \sin \alpha$ and $\sim(A-\mu \tan \alpha) h_{b} \cos \alpha$, respectively $)$ can be strongly enhanced relative to the other modes due to the large Yukawa couplings $h_{t, b}$. In addition, the $\tilde{\tilde{t}} \overline{\tilde{t}}$ and $\tilde{b} \tilde{\tilde{b}}$ modes can dominate over the $t \bar{t}$ and $b \bar{b}$ modes, respectively, in the case the $\tilde{q}$-mixing parameters $A$ and $\mu$ are large. Furthermore, in this case $\tilde{t}_{1}$ and $\tilde{b}_{1}$ tend to be light due to a large mass-splitting. Here note that the effects of the bottom Yukawa coupling $h_{b}$ and $\tilde{b}$ - mixing play a very important role for large $\tan \beta$ (see fig.2c). The reason for the dominance of the $\tilde{\tilde{t}} \overline{\tilde{t}}$ and $\tilde{\tilde{b}} \overline{\tilde{b}}$ modes in the $A^{0}$ decay is similar to that in the $H^{0}$ decay.

Quite generally, $B(H \rightarrow \tilde{\tilde{t}}, \tilde{b} \overline{\tilde{b}})$ depends on the parameters $m_{A^{0}}, M_{\tilde{Q}}, A, \mu$, $\tan \beta$ and more weakly on $M$. For a given $m_{A^{0}}$ the strongest dependence is that on $M_{\tilde{Q}}$ to which $m_{\tilde{t}}$ and $m_{\tilde{b}}$ are sensitive (see fig.1). $B(H \rightarrow \tilde{\tilde{t}}, \tilde{b} \overline{\tilde{b}})$ can be quite large in a substantial part of the parameter region kinematically allowed for the $\tilde{\tilde{t}} \bar{t}$ and $\tilde{b} \tilde{b}$ 
modes. We find that the dominance of the $\overline{t \tilde{t}}$ and $\tilde{b} \bar{b}$ modes is fairly insensitive to the assumption $M_{\tilde{Q}}=M_{\tilde{U}}=M_{\tilde{D}}=M_{\tilde{L}}=M_{\tilde{E}}$. As seen in fig. 1 the dependence on $A$ is also strong. Concerning the assumption $A_{t}=A_{b}=A_{\tau}$, we have found no significant change of $B(H \rightarrow \tilde{\tilde{t}}, \tilde{b} \tilde{\tilde{b}})\left(H=H^{0}, A^{0}\right)$ as compared to fig.2, when we take $A_{b, \tau} / A_{t}$ $= \pm 0.5, \pm 1, \pm 2$ keeping $A_{t}=A$. As $|\mu|$ increases, the dominance of the $\tilde{\tilde{t}}$ and $\tilde{b} \tilde{\tilde{b}}$ modes becomes more pronounced because of the increase of the $\left(H^{0}, A^{0}\right)$ couplings to $\tilde{\tilde{t}}$ and $\tilde{b} \tilde{\bar{b}}$. We also find that $B(H \rightarrow \tilde{\tilde{t}}, \tilde{b} \tilde{\tilde{b}}))\left(H=H^{0}, A^{0}\right)$ is nearly invariant under $(\mu, A) \rightarrow(-\mu,-A)$

Here we have not included QCD radiative corrections to the hadronic modes. It is shown in ref. 17] that both the standard QCD correction (due to gluon-quark loop) and SUSY QCD correction (due to gluino-squark loop) to the widths of $H^{0}, A^{0}$ $\rightarrow t \bar{t}, b \bar{b}$ can be large, but that the two corrections can partly or even totally cancel each other. The QCD corrections to $H^{0}, A^{0} \rightarrow \tilde{\tilde{t}}, \tilde{b} \tilde{\tilde{b}}$ are not known. It is shown in ref. [6] that the QCD corrections to the closely related process $H^{+} \rightarrow \tilde{\tilde{t}}$ are significant, but that they do not invalidate the tree-level conclusion of ref. [5] on the dominance of the $\bar{t} \bar{b}$ mode in a wide parameter region. As the structures of the couplings of $H^{0}$ and $A^{0}$ to $\tilde{t} \overline{\tilde{t}}$ and $\tilde{b} \tilde{\tilde{b}}(t \bar{t}$ and $b \bar{b})$ are similar to those of $H^{+}$to $\tilde{\tilde{t}} \overline{\tilde{b}}(t \bar{b})$, we can expect that the QCD corrections would not invalidate our tree-level conclusion on the dominance of the $\tilde{\tilde{t}}$ and $\tilde{b} \tilde{\tilde{b}}$ modes in a large parameter region.

As for the signatures of the $H^{0}$ and $A^{0}$ decays, typical $\tilde{\tilde{t}}$ and $\tilde{b} \overline{\tilde{b}}$ signals are shown in table 1 . They have to be compared with the conventional $t \bar{t}$ and $b \bar{b}$ signals, respectively, $\left(H^{0}, A^{0}\right) \rightarrow t \bar{t} \rightarrow\left(W^{+} b\right)\left(W^{-} \bar{b}\right) \rightarrow\left(f \bar{f}^{\prime}\right) b\left(f \bar{f}^{\prime}\right) \bar{b}$ (i.e., 6 jets $(\mathrm{j}$ 's), 4 j's +1 
isolated charged lepton $\left(\ell^{ \pm}\right)+$missing energy-momentum $(\not p)$, or $2 \mathrm{j}$ 's $\left.+\ell^{+} \ell^{\prime}-\not p\right)$ and $\left(H^{0}, A^{0}\right) \rightarrow b \bar{b}$ (i.e., 2 j's $)$. Note that $B\left(\tilde{t}_{1} \rightarrow c \tilde{\chi}_{1}^{0}\right) \simeq 1$ if $m_{\tilde{t}_{1}}<m_{\tilde{\chi}_{1}^{+}, \tilde{\ell}, \tilde{\nu}, \tilde{b}_{1}}$ and $m_{\tilde{\chi}_{1}^{0}}<m_{\tilde{t}_{1}}$ $<m_{\tilde{\chi}_{1}^{0}}+m_{t, W}($ in cases (a), (b) and (d) of table 1$)$, and $B\left(\tilde{t}_{1} \rightarrow c \tilde{\chi}_{1}^{0}\right) \simeq 0$ otherwise (in case (c)) [18. (In principle there is a region $m_{b}+m_{W}+m_{\tilde{\chi}_{1}^{0}}<m_{\tilde{t}_{1}}<m_{b}+m_{\tilde{\chi}_{1}^{+}}$where also the decay $\tilde{t}_{1} \rightarrow b W^{+} \tilde{\chi}_{1}^{0}$ plays a role [19]. However, this decay does not occur in the cases considered here.) As seen in table 1 , the $\tilde{t} \tilde{t}(\tilde{b} \tilde{\tilde{b}})$ signals have general features which distinguish them from the $t \bar{t}(b \bar{b})$ signals : (i) more $\not p$ due to the emission of two LSP's and hence less energy-momentum of jets and the isolated-charged-lepton in case of a short decay chain, or (ii) more jets and/or more isolated-charged-leptons in case of a larger decay chain. Moreover, depending on the values of the MSSM parameters, the $\tilde{\tilde{t}}$ and $\tilde{b} \tilde{\tilde{b}}$ signals could have the following remarkable features : (i) production of same-sign dileptons $\ell^{ \pm} \ell^{\prime \pm}$ (e.g., in case $(g)$ ), which could yield samesign isolated dilepton events such as $e^{+} e^{-} \rightarrow H^{0} Z^{0} \rightarrow\left(\ell^{+} \ell^{\prime}\right.$ or $\left.\ell^{-} \ell^{\prime}-\right)+\mathrm{j}$ 's $+\not p ;($ ii) less bottom-jet activity (e.g., in cases (a), (b), (d) with $\left(h^{0}, Z^{0}\right) \rightarrow\left(\ell^{+} \ell^{-}\right.$or $\left.\left.\nu \bar{\nu}\right)\right)$ or more bottom-jet activity (e.g., in cases (c), (d), (f) with $\left.\left(h^{0}, Z^{(*)}\right) \rightarrow b \bar{b}\right)$; and (iii) emission of a real $Z^{0}$ or $h^{0}$ (e.g., in cases (b), (c), (d), (f)). The identification of the sign of charged leptons and the tagging of $b$ - and $c$-quark jets, $h^{0}, Z^{0}$ and $W^{ \pm}$would be very useful [2] in discriminating the $\tilde{\tilde{t}}$ and $\tilde{b} \overline{\tilde{b}}$ signals from the $t \bar{t}$ and $b \bar{b}$ signals as well as in suppressing the background.

The suitable places for $H^{0}$ and $A^{0}$ search would be $e^{+} e^{-}$colliders 20] and hadron supercolliders $\llbracket$. If the $\tilde{\tilde{t}}$ and $\tilde{b} \overline{\tilde{b}}$ modes dominate the $H^{0}$ and $A^{0}$ decays, it decisively influences the signatures of $H^{0}$ and $A^{0}$. For example, it can strongly 
suppress the conventional discovery modes of $H^{0}$ and $A^{0}$ at the hadron supercollider [4], such as the "gold-plated" $H^{0} \rightarrow Z^{0} Z^{0}$ mode (viable for small $\tan \beta$ and $2 m_{Z}$ $\lesssim m_{H^{0}} \lesssim 2 m_{t}$ ) (see fig.2a) and $H^{0}, A^{0} \rightarrow \tau^{-} \tau^{+}$mode (viable for large $\tan \beta$ ) (see figs.2c, d). Clearly it would be necessary to perform a detailed Monte-Carlo study to separate the signals from the background. Such a study is, however, beyond the scope of this article.

We have shown that the SUSY modes $\tilde{\tilde{t}} \overline{\tilde{t}}$ and $\tilde{b} \bar{b}$ can dominate the $H^{0}$ and $A^{0}$ decays in a large allowed region of the MSSM parameter space due to large $t$ and $b$ quark Yukawa couplings and large $\tilde{t}$ - and $\tilde{b}$-mixings. The $\tilde{\tilde{t}} \bar{t}$ and $\tilde{b} \tilde{b}$ modes have very distinctive signatures as compared to the conventional modes $t \bar{t}, b \bar{b}$ and $\tau^{-} \tau^{+}$. This could decisively influence the $H^{0}$ and $A^{0}$ search at future $e^{+} e^{-}$and hadron colliders.

While preparing this manuscript a paper by Djouadi et al. 21] appeared dealing with a similar subject. They also point out the possible importance of the squark pair modes in $H^{0}$ and $A^{0}$ decays. They, however, studied them in a strongly constrained supergravity model in contrast to our work, which is in the general framework of the MSSM.

\section{Acknowledgements}

The authors thank Prof. V. Barger for a valuable communication on ref. 14. The work of A.B., H.E., and W.M. was supported by the "Fonds zur Förderung der wissenschaftlichen Forschung" of Austria, project no. P10843-PHY. The work of Y. Y. was supported in part by the U. S. Department of Energy under Grant No. DE- 
FG02-95ER40896 and in part by the University of Wisconsin Research Committee with funds granted by the Wisconsin Alumni Research Foundation. The work of T. K. was supported in part by the Grant-in-Aid for Scientific Research Program from the Ministry of Education, Science and Culture of Japan, No.08640388. 


\section{References}

[1] H. E. Haber and G. L. Kane, Phys. Rep. $\underline{117}$ (1985) 75.

[2] J. F. Gunion, H. E. Haber, G. L. Kane, and S. Dawson, The Higgs Hunter's Guide, Addison-Wesley (1990)

[3] J. F. Gunion and H. E. Haber, Nucl. Phys. B272 (1986) 1; $\underline{B 402}$ (1993) 567 (E).

[4] Z. Kunszt and F. Zwirner, Nucl. Phys. B385 (1992) 3.

[5] A. Bartl, K. Hidaka, Y. Kizukuri, T. Kon and W. Majerotto, Phys. Lett. B315 (1993) 360 .

[6] A. Bartl, H. Eberl, K. Hidaka, T. Kon, W. Majerotto and Y. Yamada, Phys. Lett. B373 (1996) 117.

[7] J. Ellis and S. Rudaz, Phys. Lett. B128 (1983) 248.

[8] D $\emptyset$ Collab., S. Abachi et al., Phys. Rev. Lett. $\underline{75}$ (1995) 618.

[9] E. Gallas, Proc. of the XXXIst Rencontres de Moriond, "QCD and High Energy Hadronic Interactions", Les Arcs, Savoie, France, 23-30 March, 1996, (FERMILAB-Conf-96/114-E) ; J. Conway, talk at "SUSY96", Maryland, May 1996.

[10] J. F. Grivaz, Proc. of the XXVIIIth Rencontres de Moriond on Electroweak interactions and unified theories, (Les Arcs, Savoie, France, March, 1993). 
[11] S. Asai, Proc. of the Lake Louise Winter Institute "Topics in Electroweak Physics", Alberta, 18-24 Feb. 1996 (ICEPP preprint UT-ICEPP 96-01) ; ALEPH Collab., D. Buskulic et al., Phys. Lett. B373 (1996) 246 ; L3 Collab., H. Nowak and A. Sopczak, L3 Note 1887, 1996 ; OPAL Collab., S. Asai and S. Komamiya, OPAL Physics Note PN-205, 1996 ; DELPHI Collab., DELPHI 96-101, 1996.

[12] D $\emptyset$ Collab., S. Abachi et al., Phys. Rev. Lett. $\underline{76}$ (1996) 2222.

[13] J. Ellis, G. Ridolfi and F. Zwirner, Phys. Lett. B262 (1991) 477.

[14] V. Barger, M. S. Berger, A. L. Stange and R. J. N. Phillips, Phys. Rev. D45 (1992) 4128.

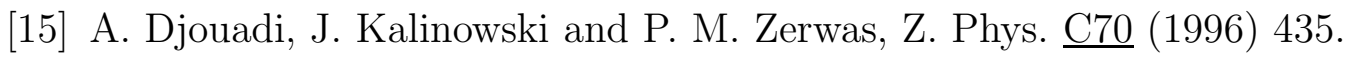

[16] Review of Particle Properties, Phys. Rev. D54 (1996) 1 ; DELPHI Collab., P. Abreu et al., Z. Phys. $\underline{\text { C67 (1995) } 69 .}$

[17] J. A. Coarasa, R. A. Jiménez and J. Solà, hep-ph/9511402 ; see also R. A. Jiménez and J. Solà, hep-ph/9511292, and A. Bartl, H. Eberl, K. Hidaka, T. Kon, W. Majerotto and Y. Yamada, Phys. Lett. B378 (1996) 167.

[18] I. I. Bigi and S. Rudaz, Phys. Lett. B153 (1985) 335 ; K. I. Hikasa and M. Kobayashi, Phys. Rev. D36 (1987) 724.

[19] W. Porod and T. Woehrmann, Vienna prprint UWThPh-1996-45. 
[20] A. Djouadi, J. Kalinowski and P. M. Zerwas, and P. Janot, Proceedings " $e^{+} e^{-}$ Collisions at 500GeV : The Physics Potential", Munich - Annecy - Hamburg 1991, ed. P. M. Zerwas, DESY report 'DESY $92-123 A^{\prime}$; A. Djouadi, J. Kalinowski and P. M. Zerwas, Z. Phys. C57 (1993) 569.

[21] A. Djouadi, J. Kalinowski, P. Ohmann and P. M. Zerwas, preprint hepph/9605339. 


\section{Figure Captions}

Fig.1 Contour lines of $B\left(H^{0} \rightarrow \tilde{\tilde{t}}, \tilde{b} \tilde{\tilde{b}}\right)(\mathrm{a}, \mathrm{c})$ and $B\left(A^{0} \rightarrow \tilde{\tilde{t}}, \tilde{b} \tilde{\tilde{b}}\right)(\mathrm{b}, \mathrm{d})$ in the $A-M_{\tilde{Q}}$ plane for $\left(m_{A^{0}}(\mathrm{GeV}), M(\mathrm{GeV}), \mu(\mathrm{GeV}), \tan \beta\right)=(450,160,300,2)(\mathrm{a}),(500$, 160, 300, 2)(b), (450, 146, 300, 12)(c) and $(500,146,300,12)(d)$. The contour of $B=0$ (dashed line) represents the kinematical boundary ; the lines (iii), (iv) and $(\mathrm{v})$ are the contour lines of $2 m_{\tilde{t}_{1}}=m_{H^{0}}(\simeq 450 \mathrm{GeV}), 2 m_{\tilde{b}_{1}}=m_{H^{0}}(\simeq 450$ $\mathrm{GeV})$ and $m_{\tilde{b}_{1}}+m_{\tilde{b}_{2}}=m_{A^{0}}(=500 \mathrm{GeV})$, respectively. The shaded area is excluded by the requirement $m_{\tilde{t}_{1}, \tilde{b}_{1}, \tilde{\ell}}>m_{\tilde{\chi}_{1}^{0}}(\simeq 70 \mathrm{GeV}) ;$ the lines (i) and (ii) are the contour lines of $m_{\tilde{t}_{1}}$ and $m_{\tilde{b}_{1}}=70 \mathrm{GeV}$, respectively, above which $m_{\tilde{t}_{1}}$ and $m_{\tilde{b}_{1}}>70 \mathrm{GeV}$.

Fig.2 The $m_{A^{0}}$ dependence of important branching ratios of the $H^{0}$ and $A^{0}$ decays for $\left(M_{\tilde{Q}}(\mathrm{GeV}), A(\mathrm{GeV}), M(\mathrm{GeV}), \mu(\mathrm{GeV}), \tan \beta\right)=(102,325,160,300,2)$ (a, b), $(218,405,145,300,30)(\mathrm{c})$ and $(145,274,146,300,12)(\mathrm{d})$. The sum over all mass eigenstates and/or flavors is taken for $B(\tilde{\tilde{t}} \overline{\tilde{t}}), B(\tilde{b} \tilde{\tilde{b}}), B\left(\tilde{\chi}^{+} \tilde{\chi}^{-}\right)$, $B\left(\tilde{\chi}^{0} \tilde{\chi}^{0}\right), B\left(\tilde{\tau}^{+} \tilde{\tau}^{-}\right)$and $B(\tilde{\nu} \overline{\tilde{\nu}})$. The shoulders in the curves for the $\tilde{\tilde{t}}$ and $\tilde{b} \tilde{\tilde{b}}$ modes correspond to opening of the $\tilde{t}_{2} \overline{\tilde{t}}_{2}$ and $\tilde{b}_{2} \overline{\tilde{b}}_{2}$ channels, respectively. 

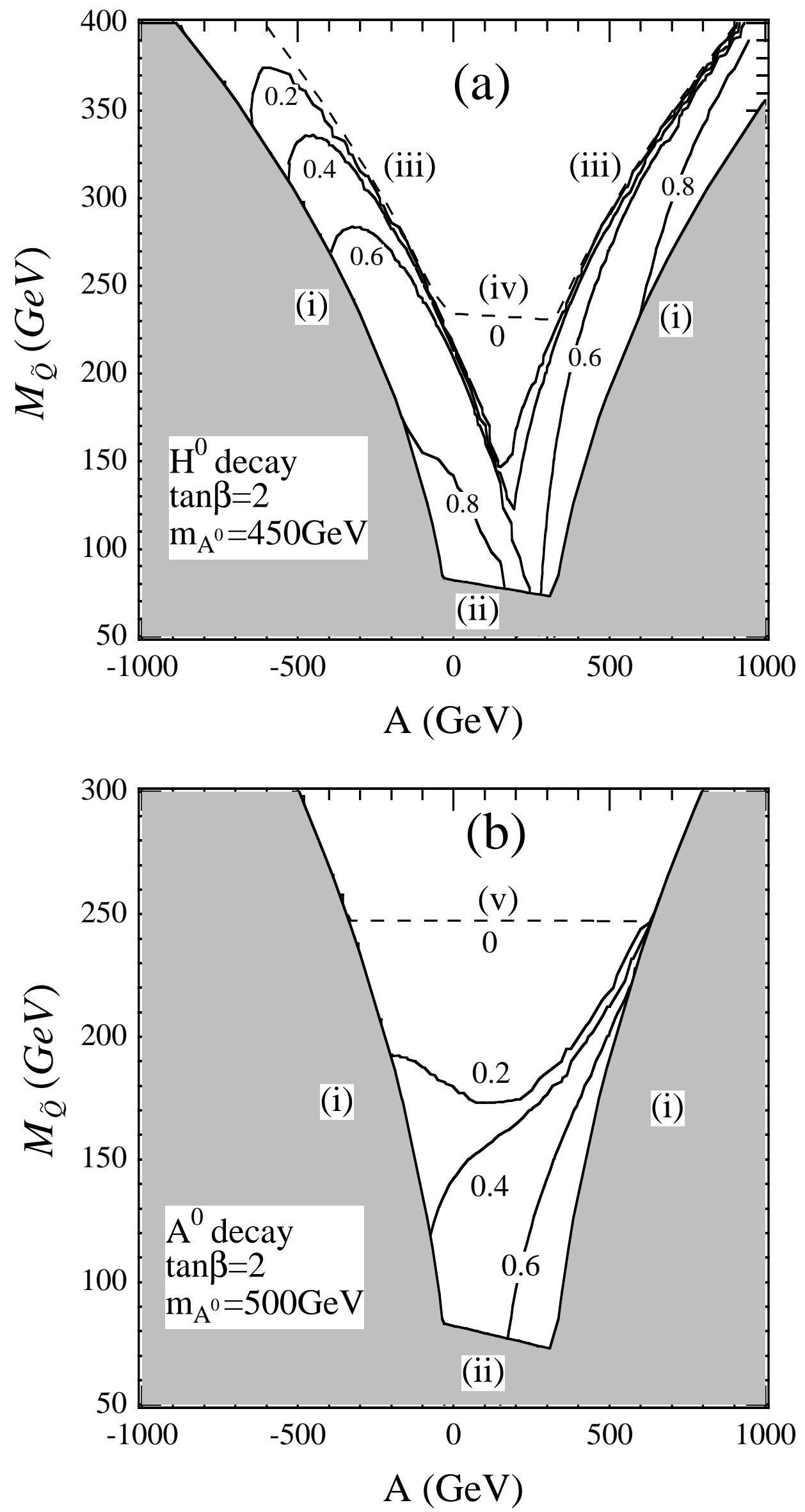

Fig.1 

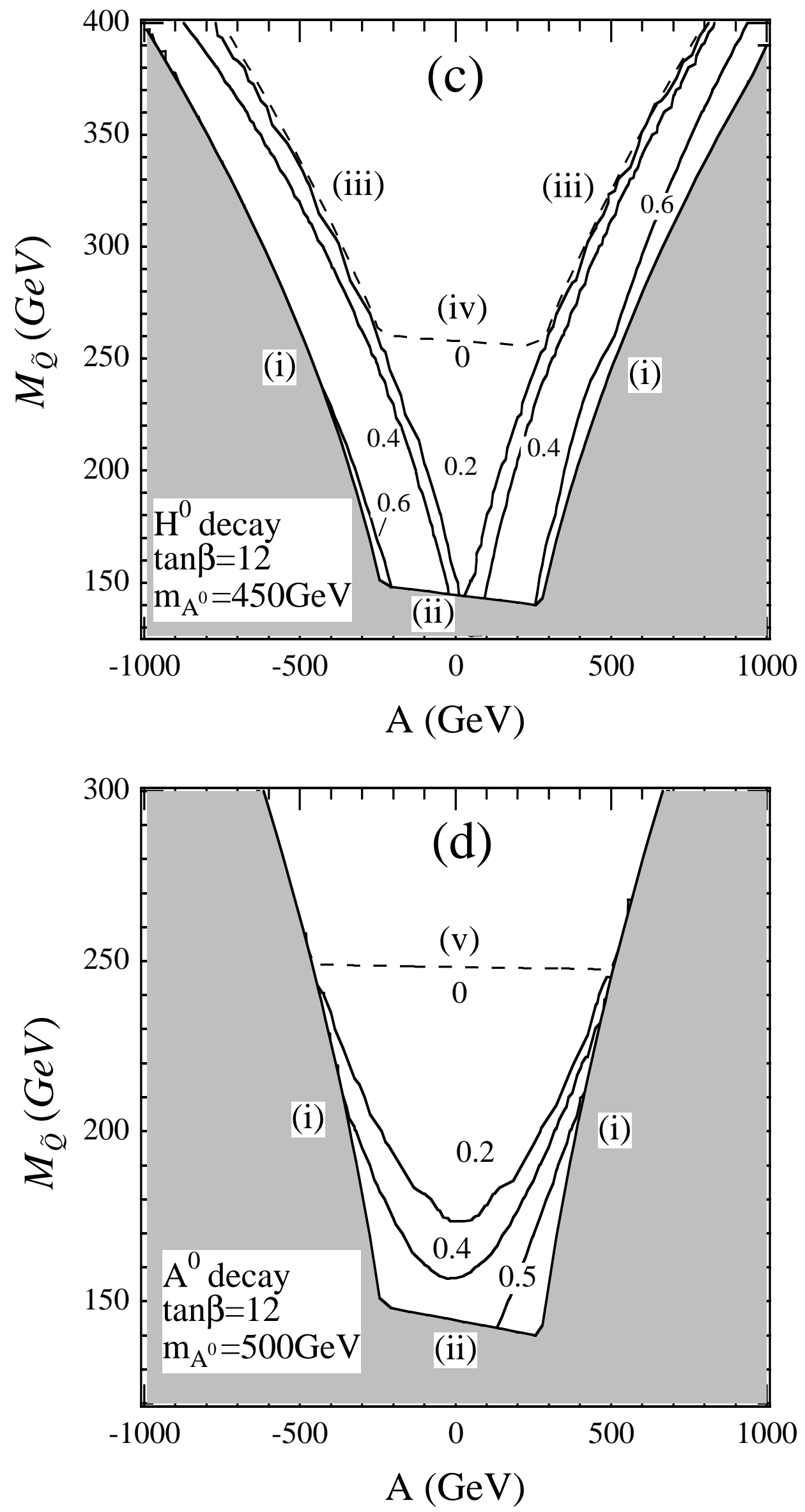

Fig.1 

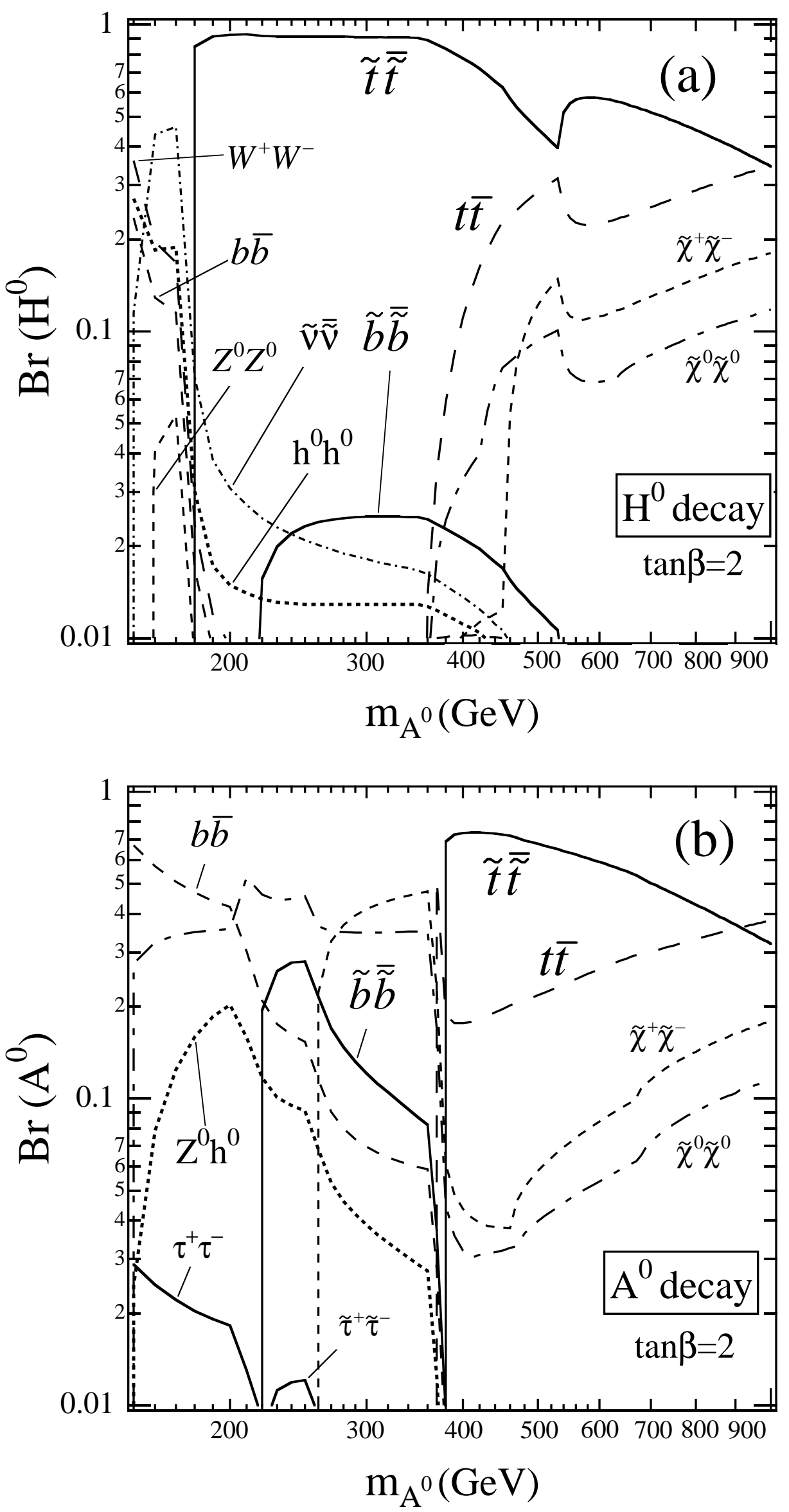

Fig.2 

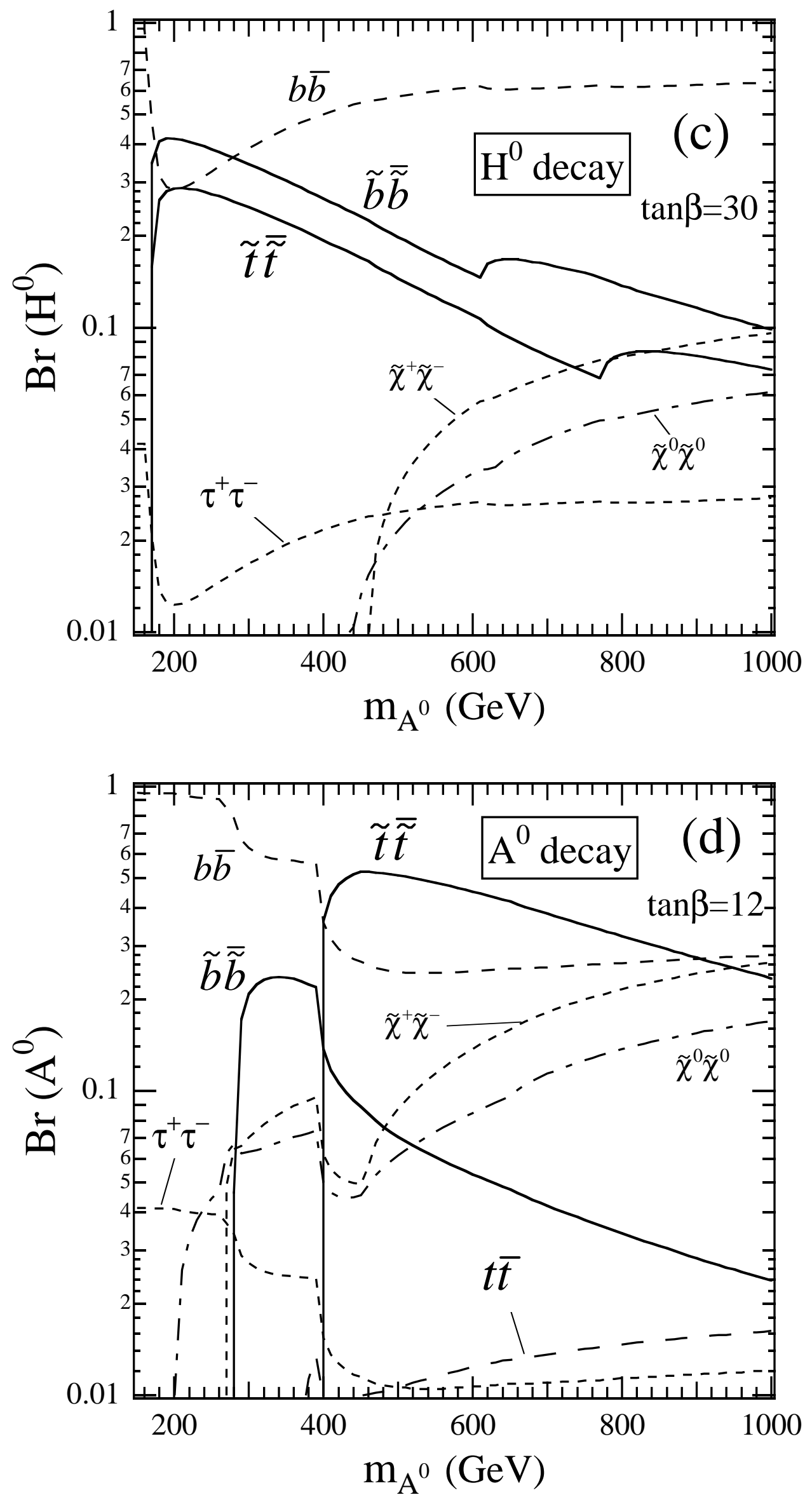

Fig.2 
Table 1: The typical $\overline{\tilde{t}}$ and $\tilde{b} \bar{b}$ signals of the $\left(H^{0}, A^{0}\right)$ decays in comparison to the conventional $t \bar{t}$ and $b \bar{b}$ signals, respectively. The decay chains (a), (b) and (d) are possible if $m_{\tilde{t}_{1}}<m_{\tilde{\chi}_{1}^{+}, \tilde{\ell}, \tilde{\nu}, \tilde{b}_{1}}$ and $m_{\tilde{\chi}_{1}^{0}}<m_{\tilde{t}_{1}}<m_{\tilde{\chi}_{1}^{0}}+m_{t, W} \cdot p, j, \ell^{ \pm}, Z^{(*)}$ and $f$ denote missing energy-momentum, jet, isolated charged lepton, real (or virtual) $Z^{0}$ boson, and $\left(q, \ell^{-}, \nu\right)$, respectively. $A^{0}$ does not couple to $\tilde{q}_{1} \overline{\tilde{q}}_{1}$ and $\tilde{q}_{2} \overline{\tilde{q}}_{2}$.

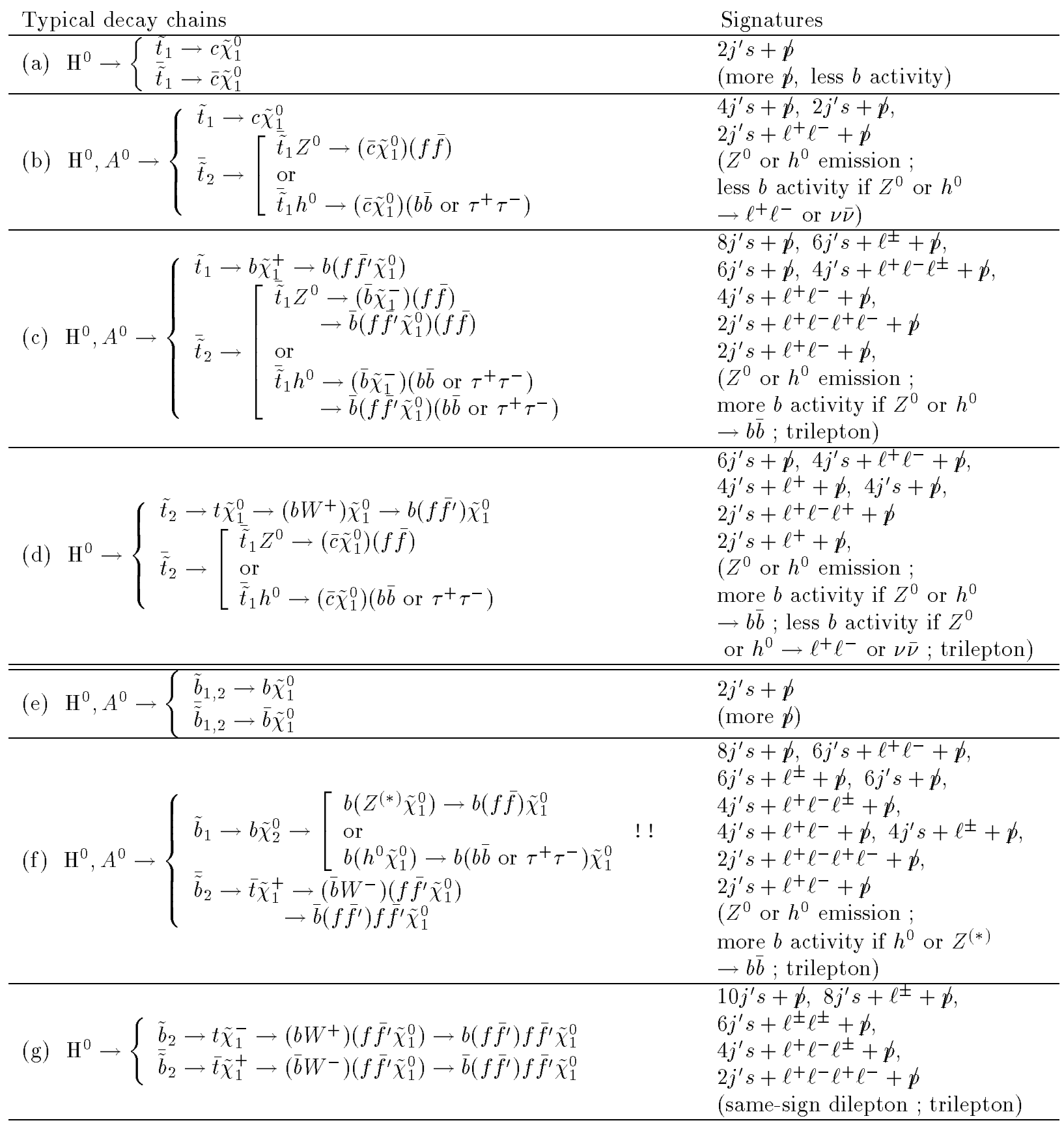

